La nature crée des formes

fascinantes comme

les « jardins chimiques»,

structures minérales

rappelant de frêles brindilles miniatures. Ces jardins,

façonnés par une réaction de précipitation, interpellent depuis des siècles par leur beauté tant admirée par le public des fêtes de la science [1]. Les jardins chimiques pourraient être une clé vers de nouvelles applications en science des matériaux, en catalyse, voire dans la compréhension de l'origine de la vie sur Terre.

Nous avons pu obtenir une grande variété

de formes de ces jardins par des réactions chimiques en milieu confiné (fig.1).

Notre étude permet de mieux comprendre la croissance de matériaux solides en condition de non-équilibre.

\title{
Mise en relief des jardins chimiques par confinement
}

Florence Haudin $^{(1,2,3)}$ (florence.haudin@espci.fr), Fabian Brau ${ }^{(1)}$ et Anne De Wit ${ }^{(1)}$

(1) Université libre de Bruxelles (ULB), Unité de Chimie Physique Non Linéaire, CP 231, Faculté des Sciences, 1050 Bruxelles, Belgique

(2) Institut Langevin, Laboratoire Ondes et Images (LOA), UMR CNRS 7587-ESPCI, 5 rue Jussieu, 75005 Paris (3) Laboratoire de Physique et Mécanique des Milieux Hétérogènes (PMMH), UMR CNRS 7636-ESPCI-UPMC-UPD, 10 rue Vauquelin, 75005 Paris

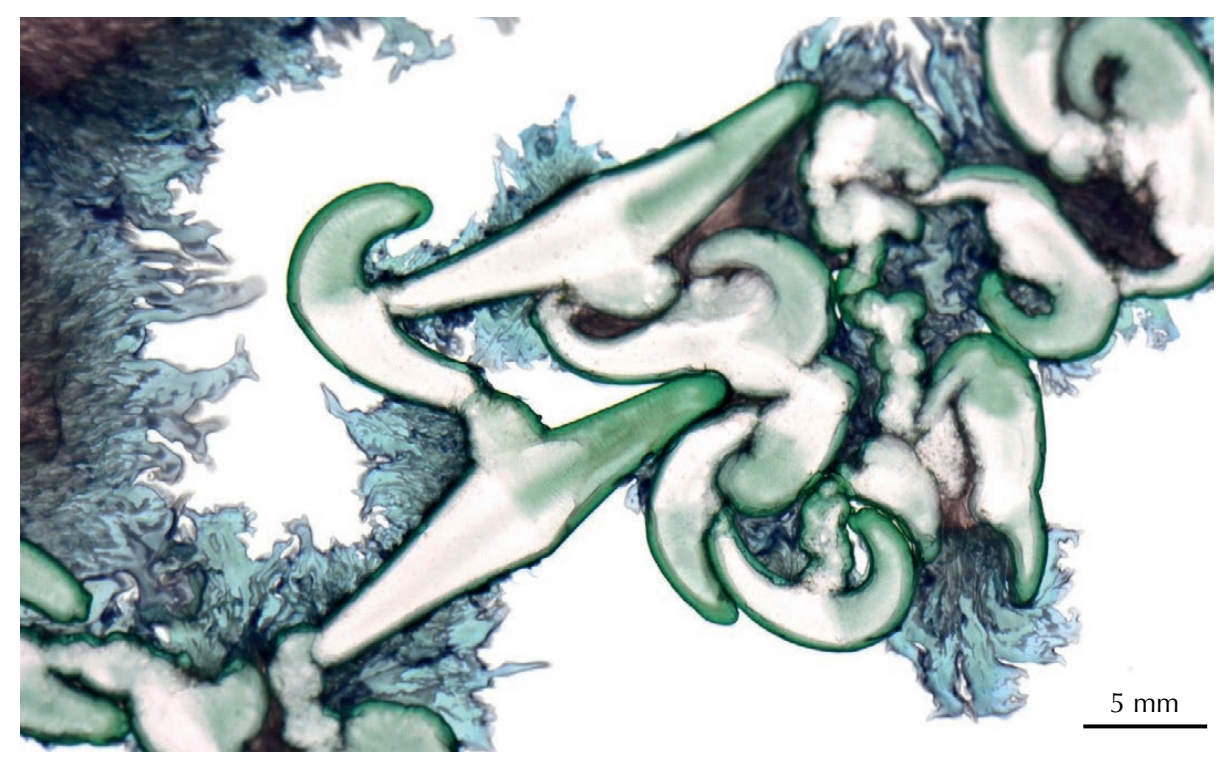

1. Un exemple de forme singulière colorée, observée dans un jardin chimique en milieu confiné. Ce type de structure, qui fait penser à des cintres ou à des boomerangs, est obtenu pour des concentrations en sel métallique intermédiaires entre celle des spirales et celle des filaments (le long de la colonne 5 du diagramme de la figure 5, p. 17).

La chimie offre de beaux exemples de structures solides créées par précipitation avec, parmi celles-ci, les spectaculaires " jardins chimiques ". On appelle réaction de précipitation une réaction dont le produit, nommé "précipité ", est un solide, amorphe ou cristallin (dans le cas d'une cristallisation). Ce solide peut, suivant sa densité par rapport à la phase aqueuse, soit rester en suspension soit se déposer au fond du récipient. Un exemple de solide se formant à partir de substances en solution est le calcul rénal, dû à la cristallisation de sels minéraux en concentration trop importante dans des urines. Si de plus, ces précipitations se couplent à un transport de matière, d'autres structures peuvent se développer. Ainsi, les stalactites sont des concrétions qui, par exemple, se forment au niveau de fissures dans la voûte d'une grotte : ils résultent de la cristallisation lente et progressive de sels, le plus souvent calcaires, provenant de la dissolution des roches.

Dans les deux exemples précédents, le processus de formation du solide est le même : la concentration en sels minéraux dépasse à un moment donné la limite de solubilité pour des conditions thermodynamiques données, si bien que les sels commencent à précipiter et l'on observe alors l'apparition d'une phase solide.

Les réactions de précipitation sont connues pour pouvoir générer des structures régulières comme, par exemple, les motifs de Liesegang consistant en des anneaux concentriques de précipité, observables sur certaines roches. Pour les obtenir en 

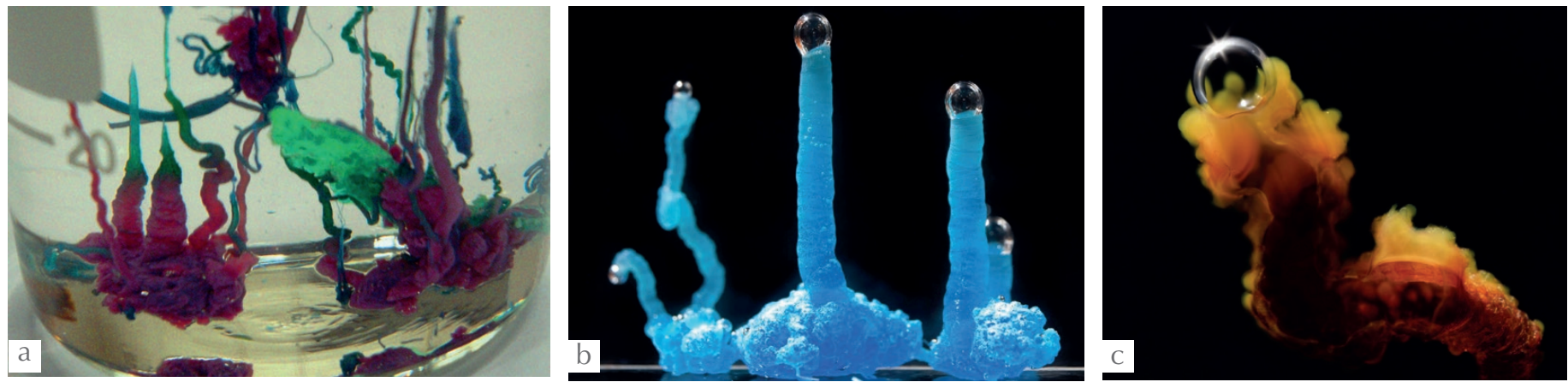

2. Photos de jardins chimiques. (a) Jardins chimiques produits dans notre laboratoire dans un simple bécher rempli d'une solution de silicate dans laquelle on a déposé des cristaux de chlorure de cobalt. (b-c) Photos artistiques de jardins chimiques réalisés avec (b) des cristaux de cuivre et (c) des cristaux de fer [S. Querbes et les Atomes Crochus, www.stephanequerbes.com/portfolio/science/, www.atomes-crochus.org/article23.html]. L'ordre de grandeur du diamètre des tubes est de quelques millimètres.

laboratoire, il suffit de verser une solution de sel métallique (tel que du nitrate d'argent, par exemple) dans un tube à essai préalablement rempli d'un gel contenant un second réactif (comme le dichromate de potassium) : la précipitation produit les premiers anneaux au bout de plusieurs heures, tandis que les suivants continuent de se former au-delà de plusieurs jours. L'utilisation d'un gel fait que le processus de formation du solide sera très lent, puisqu'il n'y a pas de mouvement de convection dans le fluide et que seule la diffusion peut agir pour mettre les réactifs en contact.

Les jardins chimiques, quant à eux, ne font pas intervenir de gel et se développent plus rapidement (sur des échelles de temps de l'ordre de l'heure). Ils sont obtenus en déposant une "graine " solide de type sel métallique dans une solution aqueuse basique à partir de laquelle des concrétions minérales commencent à " pousser " grâce à une réaction de précipitation. Ces jardins chimiques fascinent les scientifiques depuis plus de trois siècles [2] (cf. encadré 1) et sont capables de générer des formes spectaculaires, typiquement des structures tubulaires de morphologie assez complexe et de couleurs variées (fig. 2). Suivant le sel métallique utilisé ou les états d'oxydation du métal dans les produits formés, on peut obtenir un parterre de brindilles aux teintes nuancées : rose-pourpre pour le cobalt avec des tubes devenant verts ou bleus par endroits dans les zones où il y a des oxydes métalliques (fig. 2a), bleu pour le cuivre (fig. 2b), orange-rouge pour le fer (fig. 2c), blanc pour le calcium, vert pour le nickel... Voyons à présent plus en détails quels sont les mécanismes responsables de leur formation.

\section{Petit historique des jardins chimiques} encadré 1

La première observation des jardins chimiques date de 1646, suite aux travaux d'un chimiste allemand, Johann Rudolf Glauber, dont les expériences ont été source d'inspiration pour de nombreux travaux réalisés aux $17^{\mathrm{e}}$ et $18^{\mathrm{e}}$ siècles [2]. Newton lui-même y fait référence dans ses écrits "Of Nature's obvious laws and processes in vegetation", où il parle de sels métalliques et de végétation dans un "verre » (le silicate utilisé pour les jardins chimiques étant aussi appelé "verre liquide »). Au cours des $19^{\mathrm{e}}$ et $20^{\mathrm{e}}$ siècles, les études sur les jardins chimiques se sont davantage orientées vers des aspects biologiques, notamment avec les travaux du chimiste allemand Moritz Traube qui s'est attaché à comparer les structures produites par précipitation avec les cellules biologiques. Au début du $20^{\mathrm{e}}$ siècle, un nom va se démarquer dans le domaine, celui de Stéphane Leduc, biologiste et chimiste français, qui publia notamment un livre intitulé Mécanismes de la vie, où sont décrites les nombreuses croissances osmotiques qu'il a étudiées.

Vers le milieu du $20^{\mathrm{e}}$ siècle, l'idée de " créer de la vie » à partir de composés inorganiques est abandonnée avec la découverte des mécanismes génétiques et de l'ADN en 1953. Les jardins chimiques deviennent de simples exemples didactiques de structures produites par une réaction chimique.

Ils ne tombent néanmoins pas dans l'oubli et servent d'inspiration au développement de la " chimie douce » : ils seront en effet décrits par le chimiste Jacques Livage, comme une expérience " ancêtre » de cette nouvelle chimie qui a vu le jour dans les années 1970 et qui s'inspire des synthèses organiques. Son ambition est de créer de nouveaux matériaux dans des conditions thermodynamiques différentes : en remplaçant les synthèses à haute température qui génèrent des produits thermodynamiquement plus stables par des synthèses à température ambiante.

Dans les années 80, un autre élan ravive l'intérêt pour les jardins chimiques. L'accent est alors mis sur le rôle de la formation d'une membrane colloïdale entre deux solutions chimiques différentes et son lien avec des problèmes industriels tels que celui de l'hydratation du ciment de Portland (qui donne lieu à la formation de silicates de calcium hydratés) ou la corrosion, qui génère à l'air libre des structures filiformes composées d'oxydes et d'hydroxydes métalliques.

De nos jours, les recherches consacrées au sujet sont essentiellement tournées vers le contrôle de la croissance des précipités pour la synthèse de matériaux possédant certaines fonctionnalités. De plus, on suppose que les jardins chimiques possèdent des propriétés physico-chimiques assez proches de celles qui devaient exister au fond des océans primitifs où les premières formes de vie seraient apparues. Leur étude vise donc aussi à comprendre l'influence des forts gradients de composition à travers leur paroi, sur la synthèse de nouvelles molécules. Tout un domaine de recherche s'intéresse aujourd'hui à ces divers aspects des jardins chimiques. 
\〉

\section{Mécanismes de formation}

Comme décrit précédemment, un jardin chimique implique une réaction de précipitation. Les précipités formés sont constitués de particules colloïdales, assemblées en couches minces sous l'effet de plusieurs mécanismes que nous allons préciser. Une des particularités des structures solides créées dans les jardins chimiques est d'être constituées de membranes semi-perméables. Cela signifie que la membrane est perméable à certains composés mais pas à d'autres. En l'occurrence, le précipité laisse passer l'eau, le solvant, mais pas les substances qui y sont dissoutes. Nous allons voir que cette propriété est responsable en partie de l'originalité de ces structures.

Pour générer un jardin chimique, il faut mettre en contact un sel métallique, sous forme de poudre ou de pastille (donc sous forme solide), avec une solution aqueuse basique comprenant un sel de silicate, de carbonate ou de phosphate (fig. 3a). Dès que le sel est en contact avec le bain de solution alcaline, il commence dans les premières minutes à se dissoudre et à réagir avec le réactif en solution. La précipitation crée une fine membrane colloïdale de silicate de métal hydraté autour du grain solide de sel métallique (fig. 3b). Ce dernier est ainsi emprisonné dans une "coque solide " déformable, constituée d'une membrane colloïdale. Une fois que le sel commence à se dissoudre, la membrane sépare deux solutions ayant des $\mathrm{pH}$ et des concentrations très différentes. La partie extérieure de la membrane est au contact de la solution basique, tandis que la solution qui se trouve à l'intérieur est acide. L'eau

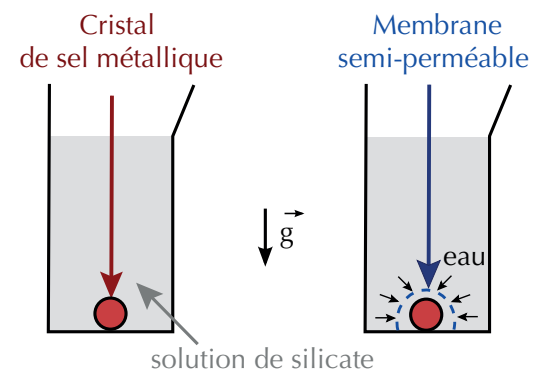

b de la solution extérieure est alors aspirée à l'intérieur de cette coque par la pression osmotique et permet à la dissolution du sel métallique de se poursuivre. Le rôle de la pression osmotique est d'homogénéiser les concentrations des solutions de part et d'autre de la membrane semi-perméable, ce qui engendre le flux d'eau mentionné. À mesure que l'eau envahit l'intérieur de la " coque solide ", la pression augmente jusqu'à rupture de la membrane, créant une brèche par laquelle la solution de sel métallique peut s'engouffrer (fig. 3c). Cette dernière, généralement moins dense que la solution extérieure de silicate, est alors expulsée vers le haut sous forme de jet sous l'effet de la poussée d'Archimède (fig. 3d). On peut imaginer de façon idéalisée que ce jet est un cylindre de quelques millimètres de diamètre, cette taille étant variable en fonction de la taille de la zone de rupture. La partie extérieure de ce cylindre est en contact avec le bain basique environnant, ce qui entraine une précipitation à mesure que le jet remonte vers la surface, d'où la formation de tubes.

En pratique, ceux-ci ne sont pas de parfaits cylindres, mais ont des formes souvent complexes et tortueuses, présentant parfois des boursouflures (fig. 2a). Du point de vue de leur structure, les parois des tubes sont constituées de plusieurs couches : à l'extérieur, une couche riche en silice (dans le cas d'un bain de silicate de sodium) et, à l'intérieur, une couche riche en oxydes ou hydroxydes métalliques. La composition des tubes est donc différente de celle de la membrane colloïdale formée initialement sur le sel métallique solide, les conditions de mise en contact des réactifs étant différentes.

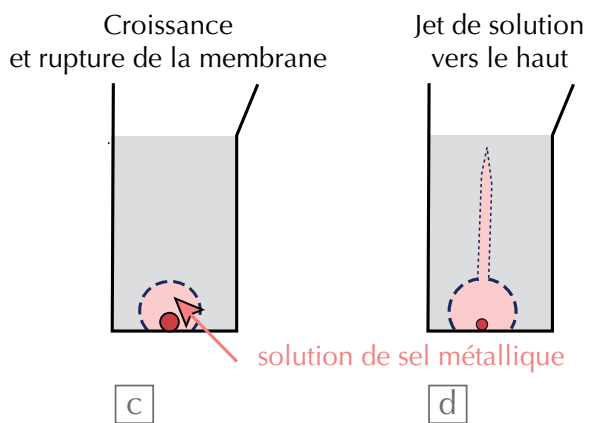

3. Différentes étapes de la formation d'un jardin chimique. (a) On place un sel métallique sous forme de sel solide, représenté ici par une sphère rouge, dans un bécher rempli d'une solution basique de silicate de sodium. (b) Une membrane semi-perméable due à la précipitation se forme autour du sel métallique. L'eau de la solution extérieure peut traverser la membrane semi-perméable et venir diluer le sel. (c) Du fait de l'apport d'eau, la pression augmente à l'intérieur de la membrane qui finit par rompre. (d) Un jet de solution de sel métallique est expulsé vers le haut, avec précipitation au contact du bain de silicate.

\section{Les jardins chimiques aujourd'hui}

Une des caractéristiques des précipités formés dans les jardins chimiques est leur croissance erratique produisant des formes irrégulières. Certains chercheurs essaient donc de mettre au point des techniques de croissance contrôlée pour maitriser la formation des structures précipitées [3]. Le but recherché est de produire des tubes de diamètre choisi ou des multicouches de composition différente. Pour mieux comprendre les dynamiques de croissance et le rôle respectif des différents phénomènes physiques et chimiques dans celle-ci (osmose, poussée d'Archimède, réaction et diffusion), des expériences où l'un des phénomènes est absent, ont été conduites. Par exemple, pour supprimer l'effet de la poussée d'Archimède, des expériences ont été réalisées en microgravité lors de vols paraboliques, avec pour résultat majeur une croissance dans des directions aléatoires et des temps d'induction beaucoup plus longs [4]. Ceci montre que la différence de densité entre la solution de sel métallique et la solution alcaline environnante constitue un véritable moteur dans la croissance verticale des structures tubulaires. Il est aussi possible d'injecter directement une solution de sel métallique dans la solution alcaline, comme dans l'étape où le jet de solution est expulsé de la membrane (fig. 3d) [5]. L'avantage est alors de pouvoir mieux contrôler la formation des précipités, puisqu'il est possible de choisir les concentrations des solutions et les débits d'injection.

Pour guider la croissance des tubes ainsi produits, différentes techniques ont été employées. Par exemple, des bulles de gaz peuvent être injectées dans le flux de solution de sel métallique, pour influencer le diamètre des tubes et guider leur croissance sous l'effet de la poussée d'Archimède. D'autres expériences ont utilisé des faisceaux laser focalisés (ou pinces optiques), dont la chaleur permet de générer un flux convectif qui dirige la croissance des tubes. Dans d'autres cas, c'est un champ magnétique qui permet de guider les dynamiques de croissance sous l'effet de la force de Lorentz exercée sur les ions en solution.

Dans tous ces exemples, les structures grandissent en volume et leur géométrie est compliquée à analyser. Pour minimiser les effets de ces inconvénients et mieux contrôler la croissance des précipités, nous avons mené des expériences avec injection de réactifs en rajoutant un effet de confinement. 


\section{Croissance en milieu confiné}

Dans notre étude, nous utilisons un dispositif constitué de deux plaques transparentes, carrées (de côté égal à 21,5 cm), horizontales et parallèles, dont l'espacement est fixé $(0,5 \mathrm{~mm})$, formant ce qu'on appelle une cellule de Hele-Shaw. Notons à titre indicatif que la distance typique entre les plaques est plus petite que l'ordre de grandeur des diamètres des tubes des jardins chimiques " classiques " (3D) décrits au paragraphe précédent. La solution basique, le silicate de sodium $\left(\mathrm{Na}_{2} \mathrm{SiO}_{3}\right)$, est placée initialement dans la cellule, tandis que la solution de sel métallique (par exemple du chlorure de cobalt $\mathrm{CoCl}_{2}$ ) est injectée radialement par un petit orifice situé au centre d'une des plaques. Comme la cellule est transparente, il est facile de filmer l'évolution des écoulements et de la précipitation au cours du temps. Pour cela, la cellule est éclairée par dessous et une caméra filme la dynamique par dessus. Une représentation schématisée du dispositif expérimental est montrée dans la figure 4. Le confinement quasi bidimensionnel imposé par le réacteur permet d'analyser plus facilement a posteriori les motifs obtenus. On peut ainsi, par exemple, mesurer l'aire des motifs ou effectuer des mesures de longueur sur leurs contours.

En concevant nos expériences, nous avions un objectif précis : caractériser les croissances obtenues en fonction des concentrations et du débit d'injection. Les expériences ont révélé une grande richesse de motifs formés par les précipités (fig. 5) [6]. Nous n'allons détailler ici que deux types de motifs : les fleurs et les spirales.

Les motifs nommés fleurs sont obtenus pour la plus grande concentration en silicate de sodium utilisée $(6,25 \mathrm{M})$ et la plus faible en sel métallique $(0,1 \mathrm{M})$; la solution déplacée est environ 40 fois plus visqueuse que la solution de sel métallique injectée. La réaction chimique et une instabilité hydrodynamique agissent alors conjointement : il s'agit d'une instabilité de digitation visqueuse, observée quand un fluide déplace un autre fluide beaucoup plus visqueux. Le précipité qui est peu cohésif est alors mis en forme par l'instabilité de digitation qui crée une structure globale en forme de fleur (fig. 5e). La concentration en sel métallique influe sur la quantité de précipité formé et sur la cohésion du précipité puisque quand on passe de 0,1 à $0,25 \mathrm{M}$ (dernière colonne du diagramme

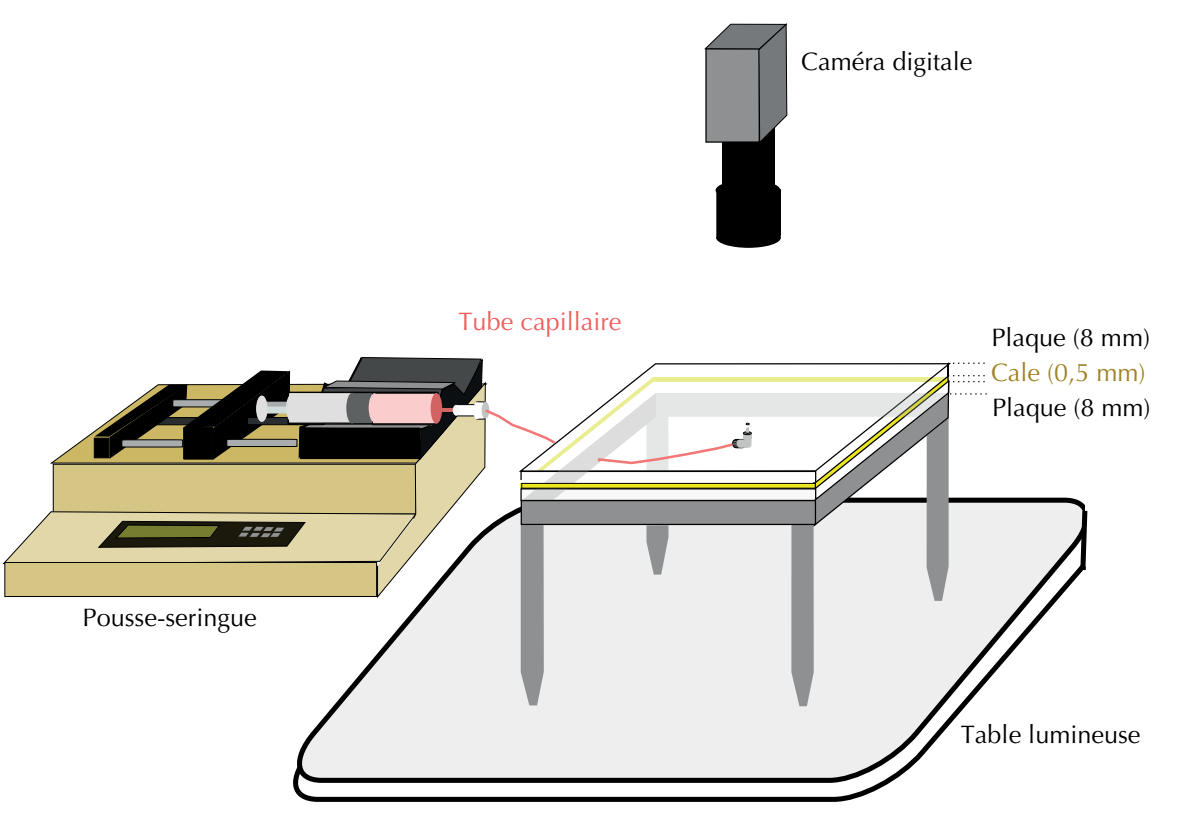

4. Schéma du dispositif expérimental utilisé pour faire croître des jardins chimiques en milieu confiné.

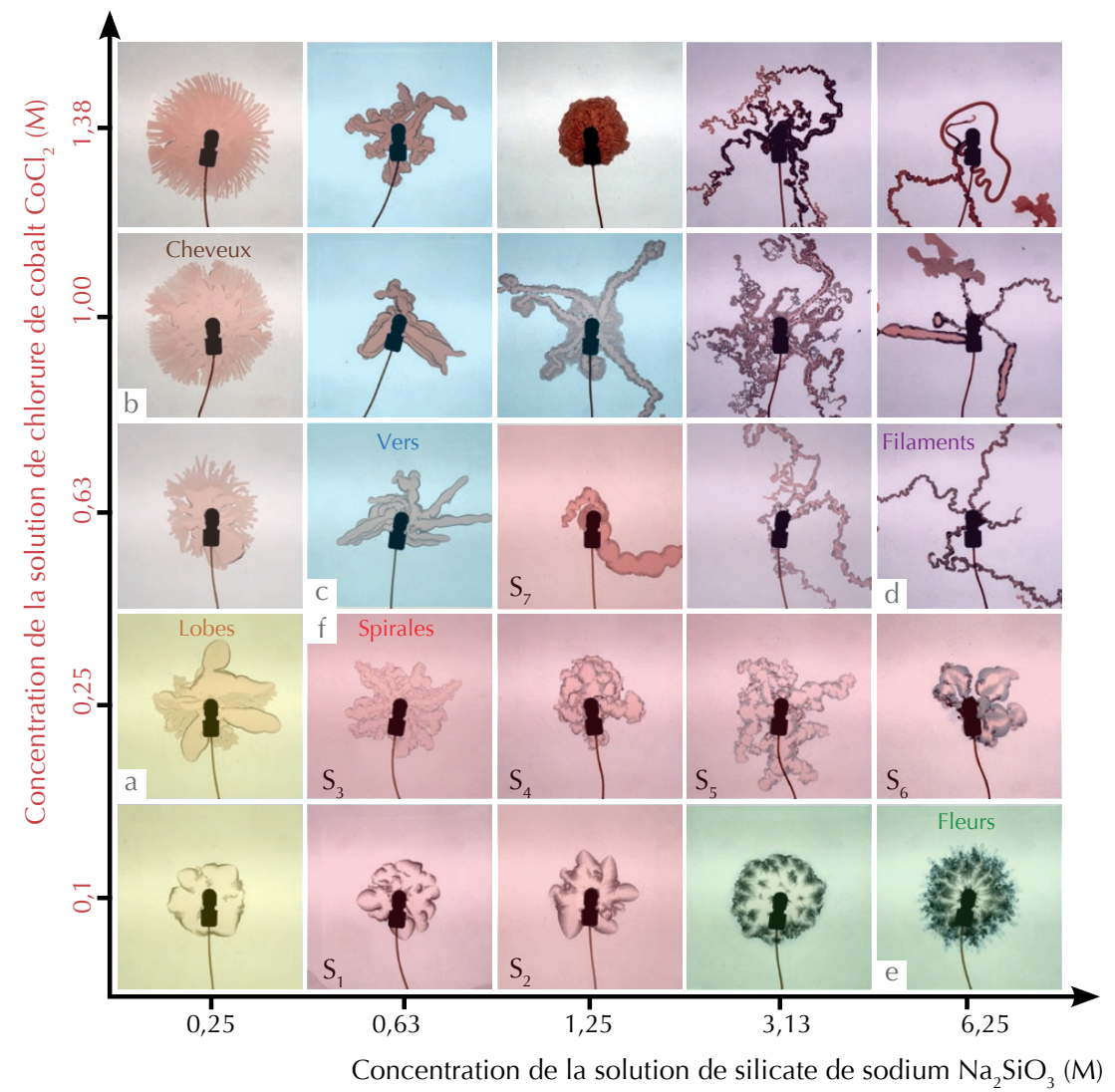

5. Différents types de jardins chimiques obtenus en géométrie confinée, observés $15 \mathrm{~s}$ après le début de l'injection de sel métallique. Les différentes couleurs permettent de localiser les différentes catégories de structures : Iobes (a, jaune), cheveux (b, gris), vers (c, bleu), filaments (d, violet), fleurs (e, vert), spirales (f, rose). Les images indexées $S_{i}$ correspondent à des couples de concentrations pour lesquels des précipités en forme de spirales sont observés et analysés sur la courbe de la figure 6d. Notons que les spirales ne sont clairement discernables que si on "zoome " suffisamment sur une région donnée, ces structures faisant en moyenne quelques mm à quelques $\mathrm{cm}$ et le champ de vue de chaque image étant de $15 \mathrm{~cm}$ de côté. 


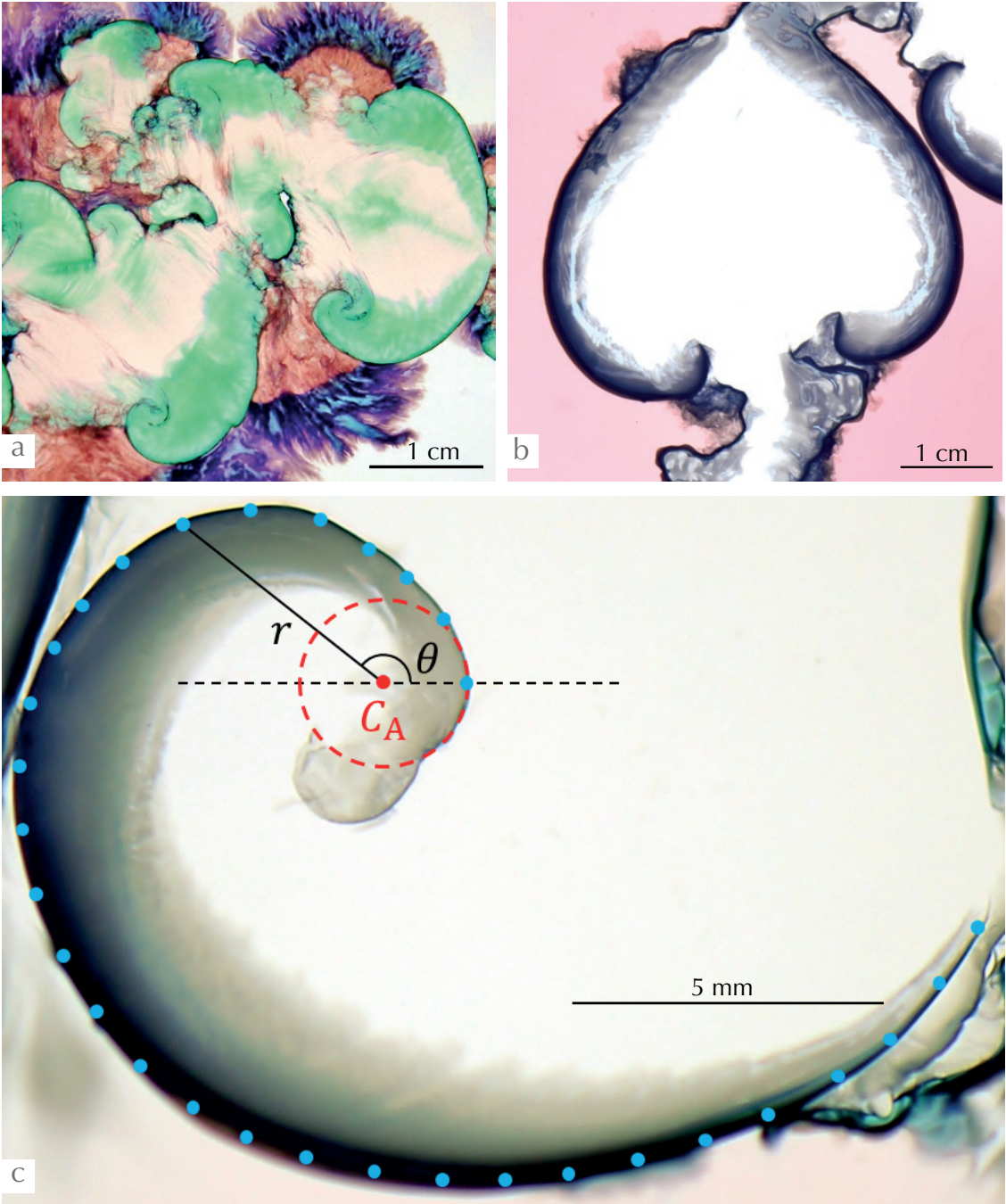

À partir des points bleus, on mesure et on trace $r$ en fonction de $\theta$. On trouve une croissance exponentielle $: r=r_{0} e^{\theta / \theta_{0}}$ (spirale logarithmique). Par régression, on peut déterminer $r_{0}$ et $\theta_{0}$.

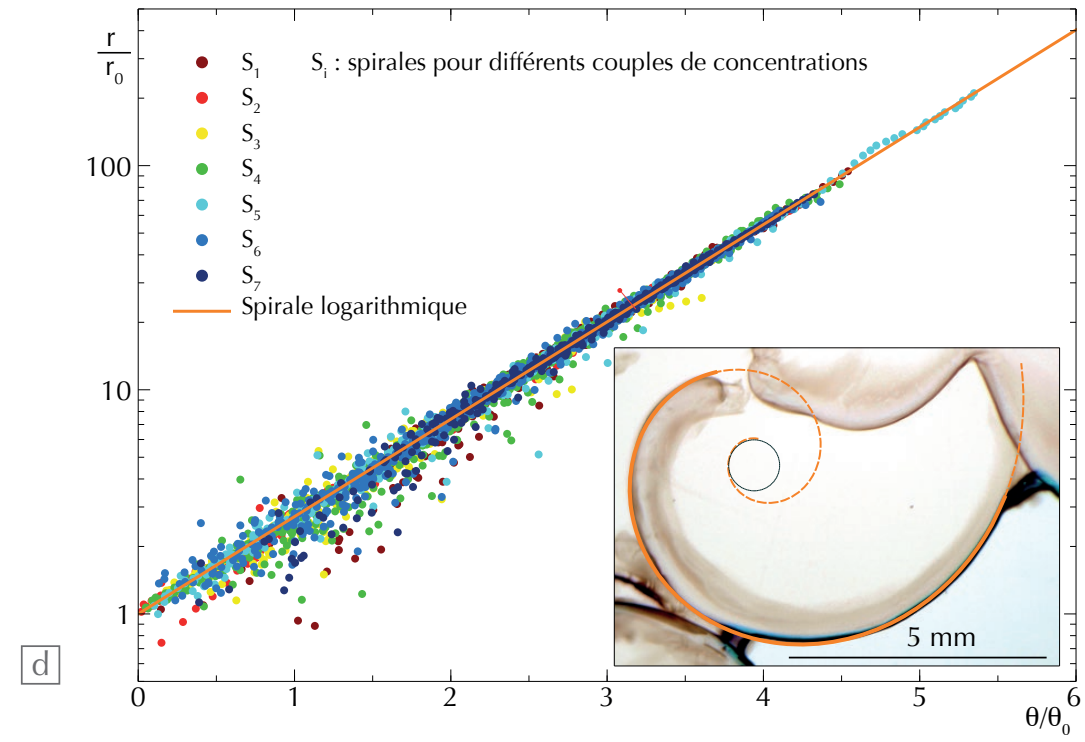

6. Précipités en forme de spirale. (a-b) Exemples de motifs spiralés. (c) Schéma expliquant comment on analyse la forme des précipités: la distance $r$ par rapport à un centre est mesurée en fonction de l'angle $\theta$ pour différents points du contour, matérialisés ici en bleu. (d) Mesures pour les spirales expérimentales de $r / r_{0}$ en fonction de $\theta / \theta_{0}$ en échelle semi-logarithmique. Les différentes catégories de spirales notées $S_{i}$ correspondent à différents couples de concentrations indiqués sur le diagramme de la figure 5 .

\section{\>}

de la figure 5), on passe d'une fleur à des spirales. Au-delà d'un certain seuil de concentration en chlorure de cobalt (autour de 0,5 M), les spirales deviennent des filaments qui sont insensibles au saut de viscosité entre les deux solutions réactives.

Les spirales sont obtenues pour toute une gamme de concentrations intermédiaires (fig. 5f). Une attention particulière leur a été portée : des photographies zoomées sont montrées sur les figures $6 \mathrm{a}, \mathrm{b}, \mathrm{c}$. Ces structures sont aussi formées dans le cas des jardins chimiques dits inversés, c'est-à-dire dans le cas où on injecte la solution alcaline dans celle de sel métallique. Tous les cas où la solution déplacée est colorée (rose à l'extérieur des précipités spiralés) correspondent à cette situation (exemples sur les figures $6 \mathrm{~b}-\mathrm{c}$ et $7 \mathrm{~g}$ ). La formation des spirales peut être expliquée à l'aide d'un modèle simple, prédisant l'apparition de spirales logarithmiques (encadré 2). Pour confronter la réalité des expériences à ce modèle, un ensemble d'environ 170 précipités spiralés a été analysé d'un point de vue géométrique, comme expliqué sur la figure 6c. Une loi d'échelle, caractéristique des spirales logarithmiques, a été mise en évidence, montrant un caractère universel des formes de ces précipités qui apparaissent de façon robuste pour différents couples de concentrations (fig. 6d).

\section{Perspectives}

Notre étude, en abordant les croissances de phases solides en milieu confiné d'un point de vue dynamique à l'échelle macroscopique, a révélé un grand nombre de structures différentes, lorsque l'on fait varier la concentration des réactifs utilisés et la vitesse d'injection. Elle montre que l'injection en milieu confiné est un moyen prometteur pour caractériser les divers types de croissance possible de précipités dans un système maintenu hors d'équilibre. Cependant, pour mieux comprendre la dynamique à l'échelle macroscopique, il sera aussi utile d'étudier l'influence des conditions hors d'équilibre sur les structures microscopiques, pour déterminer en particulier comment les flux liés à l'injection agissent localement sur la précipitation et comment ceci se répercute à plus grande échelle.

De plus, une caractérisation des propriétés chimiques et mécaniques des précipités aidera dans le futur à mieux expliquer ce qui régit la croissance des solides en présence 
d'un flux hydrodynamique, et comment notre technique d'injection pourrait être utilisée pour contrôler la nature et les propriétés des substances solides formées. Ces expériences ouvrent donc la voie vers le contrôle de la production de structures solides par précipitation sous injection en milieu confiné. Ce contrôle pourra tirer parti du vaste espace des paramètres disponible dans ces expériences puisque les concentrations, les vitesses d'injection et la taille du confinement peuvent être variées. De plus, en alternant l'injection de réactifs de nature différente, des structures à multicouches pourraient être synthétisées, permettant ainsi de produire de nouveaux catalyseurs ou des matériaux aux propriétés anisotropes.

\section{Références}

1• F. Haudin, F. Brau, J. Cartwright et A. De Wit, "Les nouvelles variétés des jardins chimiques confinés", La Recherche 512 (2016) 53.

2- J.R. Glauber, Furni Novi Philosophici (1646), Fabel, Amsterdam.

3• L.M. Barge et al., "From chemical gardens to chemiobrionics", Chemical Reviews 115 (2015) 8652.

4 J.H.E. Cartwright, B. Escribano, C.T. Sainz-Diaz et L.S. Stodieck, "Chemical-garden formation, morphology and composition. II. Chemical gardens in microgravity", Langmuir 27 (2011) 3294.

$5 \bullet S$. Thouvenel-Romans et 0. Steinbock, "Oscillatory growth of silica tubes in chemical gardens”, J. Am. Chem. Soc. 125 (2003) 4338.

6• F. Haudin, J.H.E. Cartwright, F. Brau et A. De Wit, "Spiral précipitation patterns in confined chemical gardens", Proc. Nat. Acad. Sci. 111 (2014) $17363-17367$.

\section{Modèle géométrique des spirales} encadré 2

Le modèle suppose qu'initialement une " goutte » de solution de sel métallique croît dans la solution de silicate : il se forme une couche de précipité dans la zone de contact entre les deux réactifs (fig. 7a). L'injection se fait de façon continue, via un point d'injection $I$. (Notons que le modèle fait l'hypothèse idéalisée d'une " coque » de précipité qui croît de façon isolée, mais dans les expériences les protubérances formées par deux spirales comme décrit sur la figure $7 \mathrm{e}$, se forment sur une embase de précipité préexistant, comme illustré sur la figure $7 \mathrm{~g}$ ). La croissance de la " coque » se poursuit jusqu'à un rayon critique $r_{C^{\prime}}$ au-delà duquel elle casse (fig. 7b). La figure 7c montre la coque de précipité avec deux cassures. Entre l'instant $t$ de la rupture et un instant ultérieur très proche $t+d t$, les deux fluides se retrouvent à nouveau en contact au niveau de l'extrémité (point $P_{0}$ ) et un ajout de matière de longueur $d S$ a lieu, pendant que la structure continue de grandir et de tourner d'un petit angle $d \theta$ (fig. $7 \mathrm{~d}$ ). Si on considère par simplicité seulement la partie droite de la structure, le point de rupture qui se trouvait au point $P_{0}$ au temps $t$ se trouve au point $P_{1}$ à l'instant $t+d t$ : il s'est décalé de $d S$ et a subi une rotation de $d \theta$ en raison de l'injection. Pour la partie gauche, la rotation est $d^{\prime}$ angle $-d \theta$. Les courbes violette et orange ont la même forme globale, mais la courbe orange s'est allongée de $d S$ et a tourné de $d \theta$ (fig. 7e). Au final, ce type de croissance donne lieu à une spirale logarithmique. Cette croissance est auto-similaire, dans la mesure où elle préserve la forme globale du précipité : la longueur croît au cours du temps par ajouts successifs de matière à une des extrémités. Les équations associées à $d S$ puis à l'évolution de $r$ en fonction de $\theta$ sont posées dans la figure $7 f$.

On retrouve ce type de croissance logarithmique dans d'autres structures naturelles, comme les coquillages ou certaines cornes d'animaux. Notons que la partie appelée extrémité est la seule zone de contact entre les deux réactifs et constitue par conséquent le seul endroit où de la matière solide peut être générée, comme on peut le voir sur la séquence $\mathrm{d}^{\prime}$ images expérimentales de la figure $7 \mathrm{~g}$ (cas d'un jardin chimique inversé, où la solution de silicate est injectée dans la solution de sel métallique). En pratique, dans I'expérience, on n'observe pas une cassure nette au niveau du point $P_{0}$ à l'instant $t$, mais une zone plus fragile et plus fine où la création de matière est possible. Au niveau de la pointe, on n'a qu'un " jet » de solution injectée et pas de contact avec le second réactif.

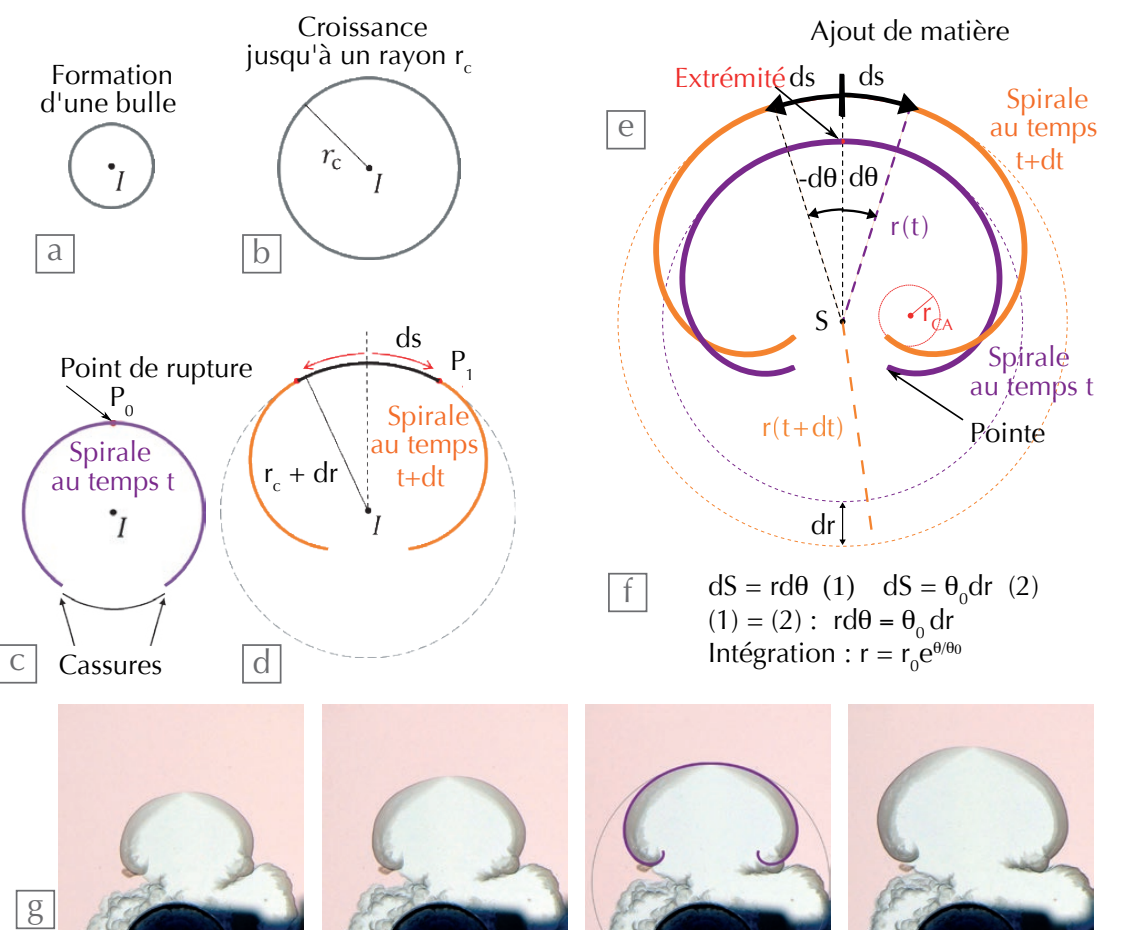

7. Formation et croissance d'un précipité en forme de spirale.

(a à d) Étapes permettant de comprendre la construction du modèle. / est le point d'injection.

(e) Schéma avec les courbes décrivant les spirales aux instants $t$ et $t+d t$.

(f) Mise en équation du modèle avec $\theta_{0}$ le taux de croissance des précipités (plus $\theta_{0}$ est grand, plus les spirales sont enroulées).

(g) Séquence d'images expérimentales montrant la croissance d'un précipité en forme de spirale dans le cas d'un jardin chimique inversé. Le bon accord avec les spirales logarithmiques est mis en évidence par les contours violets. 